\title{
Validade concorrente entre instrumentos de avaliação da compreensão em leitura e da escrita
}

\author{
Concurrent validity between assessment instruments in reading comprehension and writing
}

\author{
Adriana Cristina Boulhoça Suehiro ${ }^{[a]}$, Acácia Aparecida Angeli dos Santos ${ }^{[b]}$
}

\footnotetext{
${ }^{[a]}$ Psicóloga, Doutora em Psicologia pelo Programa de Pós-Graduação Stricto Sensu em Psicologia da Universidade São Francisco e docente da Universidade Federal do Recôncavo da Bahia - Centro de Ciências da Saúde /Santo Antônio de Jesus, BA - Brasil, e-mail:dricbs@yahoo.com.br. Apoio financeiro - Capes.

${ }^{[b]}$ Psicóloga, Doutora em Psicologia Escolar e Desenvolvimento Humano pela USP e docente da Graduação e do Programa de Pós-Graduação Stricto Sensu em Psicologia da Universidade São Francisco/ Itatiba, Bolsista produtividade do CNPq.
}

Recebido: 02/02/2010 Received: 02/02/2010

Aprovado: 03/11/2010 Approved: 11/03/2010

\section{Resumo}

O presente estudo buscou por evidência de validade concorrente entre instrumentos de avaliação da compreensão em leitura e da aprendizagem da escrita. Participaram 221 crianças, ambos os sexos, entre 6 e 12 anos $(M=8,53$; DP = 1,40) de primeira a quarta séries do ensino fundamental de uma escola pública do interior de São Paulo. A coleta de dados foi coletiva, tendo sido os participantes submetidos ao teste de Cloze, elaborado para avaliação da compreensão em leitura de alunos do ensino fundamental, e à Escala de Avaliação da Escrita (EAVE). Assumindo que as habilidades de leitura e escrita envolvam processos distintos, os resultados obtidos confirmaram a estreita relação existente entre ambas, ilustrada pelo alto índice de correlação identificado entre as duas medidas ( $\mathrm{r}=-0,84 ; \mathrm{p}<0,001)$, o que lhes confere a evidência de validade pretendida. Ao lado disso, ficou demonstrado que o nível escolar e a idade das crianças são variáveis importantes na avaliação da compreensão em leitura e da aprendizagem da escrita.

Palavras-chave: Aprendizagem. Escrita. Cloze. Leitura e escrita.

\section{Abstract}

This study aimed at searching for concurrent validity evidence between instruments assessing reading comprehension and writing learning. Participants were 221 children, of both genders, ages ranging from 6 to 12 years old $(M=8.53$; $S D=1.40)$, who attended first to fourth grades of elementary level from a public school located in the interior of São Paulo state. Data colletion was made collectively, having participants been submitted to Escala de Avaliação da Escrita (EAVE) test and to Cloze Test, which was created to assess reading comprehension in elementary school students. Assuming that writing and reading abilities involve distinct processes, the results obtained confirmed the close relationship between both of them, which is illustrated by the high correlation index that was identified between both measures ( $r=-0.84 ; p<0.001)$, what conferred the expected evidence of valitity to those measures. Besides, it was showed that children schooling and age group are important variables when assessing reading comprehension and writing learning.

Keywords: Learning. Writing. Cloze. Reading and writing. 


\section{Introdução}

A leitura e a escrita são temáticas que têm despertado o interesse de estudiosos de diferentes áreas em razão da sua importância, especialmente no mundo moderno. De acordo com Santos (1994), essas habilidades têm sido compreendidas a partir de uma visão integradora desde meados da década de 1980 . No entanto, há que se ressaltar que a aprendizagem dessas habilidades instrumentais envolve particularidades e, portanto, processos distintos. A leitura parte da informação visual e chega ao som, envolvendo a decodificação, enquanto a escrita se inicia a partir dos segmentos fonológicos e da associação dos fonemas às letras constituindo-se, assim, num processo que abarca predominantemente a codificação (Capovilla \& Capovilla, 2000; Coll, Palácios \& Marchesi, 1995; Garcia, 1998; Salles \& Parente, 2002).

Considerando-se a complexidade de tais construtos, bem como a gama de variáveis que podem interferir no processo de compreensão da leitura e da aprendizagem da escrita, tais como sua modificação em função da idade, série e sexo das crianças, ressalta-se a importância de se compreender os fundamentos teóricos que subsidiam os instrumentos que se prestam a realizar uma estimativa do nível de compreensão em leitura e da aprendizagem da escrita dos alunos e a identificar as dificuldades específicas nos comportamentos de ler e escrever (Bradley \& Bryant, 1983; Capovilla, Dias \& Montiel, 2007; Cunha, Suehiro, Oliveira, Pacanaro \& Santos, 2009; Maluf \& Barrera, 1997; Oliveira, Boruchovitch \& Santos, 2007; Pestun, 2005; Sisto, 2001; Suehiro \& Santos, 2005).

Ao lado disso, no que se refere à compreensão em leitura, destaca-se que não é fácil mensurá-la, uma vez que ela não é diretamente observável. Somando-se a isso, há o fato de que os métodos geralmente utilizados para a avaliação da compreensão são pautados em procedimentos que não garantem sua fidedignidade e que, na maioria das vezes, revelam viéses por parte do avaliador, seja pela precariedade dos instrumentos utilizados no Brasil, seja pelo uso indiscriminado de materiais de avaliação estrangeiros sem que haja preocupação com o seu sentido e validade (Noronha, 2002; Sisto, Codenotti, Costa \& Nascimento, 1979).

Nesse sentido, deve-se considerar outro tipo de viés com relação aos procedimentos e instrumentos empregados, qual seja, o que pode ser causado por problemas relacionados à sensibilidade da medida utilizada. 0 trabalho de Sousa (2005) com 31 crianças da primeira série do ensino fundamental, especificamente no que se refere à compreensão em leitura, é um exemplo claro deste viés. A fim de verificar as relações existentes entre habilidades sintáticas implícitas e explícitas e leitura sob a forma de reconhecimento de palavras e compreensão de frases, a autora utilizou uma tarefa de compreensão que pode ser considerada como muito fácil, visto que $60 \%$ dos participantes obtiveram escores altos, o que pode ter potencializado os resultados que dependiam dessa habilidade.

Daí a necessidade de instrumentos que realmente sejam válidos e confiáveis a fim de que se faça um diagnóstico preciso da habilidade dos estudantes e se implante estratégias adequadas à recuperação das deficiências detectadas. Um dos instrumentos que tem sido empregado como técnica de diagnóstico e de remediação da compreensão em leitura e considerado confiável na avaliação dessa habilidade é a técnica ou teste de Cloze (Santos, 2005, Oliveira, Boruchovich \& Santos, 2009).

A técnica de Cloze foi desenvolvida por Taylor, em 1953, e consiste na organização de um texto, do qual se omitem alguns vocábulos e se pede ao leitor que, após sua leitura integral, preencha as lacunas com as palavras que melhor completarem o sentido do texto. A alta aceitação da técnica tem sido demonstrada pelas inúmeras publicações discorrendo sobre o leque de possibilidades de utilização desse recurso (Abraham \& Chapelle, 1992; Oliveira, Boruchovitch \& Santos, 2009).

Considerada como um procedimento simples, flexível, que não coloca intermediários entre o leitor e o texto, a técnica de Cloze, como meio de avaliação da compreensão da leitura, apresenta, ainda, outras vantagens. Uma delas é o fato do leitor contar com o contexto como único apoio e não se correr o risco de que ele use palavras chave da pergunta para adivinhar a resposta a ser dada, evitando, dessa forma, a utilização de perguntas cuja compreensão poderia ser mais difícil do que a própria leitura. Outra vantagem está relacionada à utilização da técnica como um meio de desenvolvimento da compreensão da leitura, ao permitir ao aluno adivinhar as palavras que se encontram omitidas no texto, graças ao seu domínio das estruturas semântico-sintáticas da linguagem e de seus conhecimentos anteriores acerca do conteúdo do texto (Bitar, 1989; Giordano, 1985; Santos, Boruchovitch \& Oliveira, 2009). 
Dentre os estudos realizados com a população adolescente e infantil, a maioria tem se dedicado ao processo de remediação em detrimento de investigações que verifiquem a compreensão em leitura e atestem a validade do instrumento para a avaliação dessa habilidade, possibilitando o uso adequado da técnica e das informações que dela proveem e garantindo-lhe maior legitimidade. De modo geral esses estudos têm evidenciado que a técnica de Cloze é um instrumento que possibilita, de maneira eficiente, o treinamento em habilidades que favorecem a compreensão em leitura (Carelli, 1992; Joly \& Lomônaco, 2003; Santos, 2004).

Independentemente da amostra focalizada, alguns autores têm ressaltado que análises sobre a técnica permitem afirmar que ela é mais que um instrumento de avaliação da leitura, pois em várias de suas formas possibilita a avaliação também da escrita. Fato este que, se por um lado, pode ser visto como um aspecto favorável em razão da estreita relação entre os processos de leitura e escrita (Abraham \& Chapelle, 1992; Ashby-Davis, 1985; Poersch, 1993; Zucoloto \& Sisto, 2002), por outro, pode ser muito negativo e até considerado como um problema.

Psicometricamente, o fato do Cloze possibilitar também a avaliação da escrita é considerado um problema, porque, contrariando sua validade, o mesmo instrumento estaria medindo dois construtos diferentes, haja vista que ambos apresentam suas especificidades. A leitura parte, conforme ressaltado anteriormente, da informação visual e chega ao som, enquanto que a escrita associa fonemas a letras abarcando, desse modo, processos diferentes (Capovilla \& Capovilla, 2000; Garcia, 1998; Santos, 1994; Salles \& Parente, 2002).

A relação estreita entre os processos de leitura e escrita tem sido destacada por vários autores, dentre os quais Zucoloto e Sisto (2002), Santos, Sampaio, Lukjanenko, Cunha e Zenorini (2006) e Cunha (2006). Esses estudos serão descritos, resumidamente, a seguir, uma vez que o teste de escrita aqui utilizado, a Escala de Avaliação da Escrita (EAVE), foi derivado da escala por eles empregada.

A compreensão em leitura de crianças com dificuldade de aprendizagem da escrita foi investigada por Zucoloto e Sisto (2002) em 194 crianças de segunda e terceiras séries, de uma escola pública de periferia. Os níveis de dificuldade de aprendizagem da escrita foram categorizados segundo a Escala de Avaliação de Dificuldades de Aprendizagem da Escrita (Adape) e o nível de compreensão em leitura avaliado por dois textos estruturados de acordo com a técnica de Cloze. Os resultados mostraram que na segunda e terceira séries os erros na compreensão da leitura aumentavam em razão da dificuldade de aprendizagem da escrita e que os mais velhos apresentavam mais erros do que os mais novos, sendo que as análises por sexo não indicaram diferenças significativas.

A relação entre as dificuldades em compreensão em leitura e escrita também foi verificada por Santos et al. (2006). As autoras aplicaram um texto em Cloze e o Adape em 512 estudantes de segunda a quarta séries de seis escolas particulares e onze públicas do Estado de São Paulo. Diferentemente do observado por Zucoloto e Sisto (2002), os resultados evidenciaram diferenças estatisticamente significativas entre os sexos, sendo que as mulheres obtiveram um melhor desempenho quando comparadas aos homens, bem como que os alunos de escolas particulares se saíram significativamente melhor que os de escola pública em ambos os instrumentos, sendo que somente o Adape diferenciou a segunda e a quarta séries. Por fim, as autoras observaram que os escores do Adape (expressos pelo número de erros) se correlacionaram negativamente com os escores do Cloze (número de acertos), o que vai ao encontro da hipótese de trabalho dos autores.

Do mesmo modo, Cunha (2006) encontrou diferenças significativas entre os sexos em todos os instrumentos por ela empregados, a saber, Escala de Avaliação de Dificuldades de Aprendizagem da Escrita (Adape), Escala de Reconhecimento de Palavras e o teste de Cloze, sendo que as mulheres apresentaram desempenhos superiores aos homens. A autora realizou um estudo com 266 crianças, ambos os sexos, entre 8 e 13 anos de terceiras e quartas séries do ensino fundamental de escolas públicas e particulares do interior de São Paulo. Os alunos de escola particular também apresentaram escores significativamente superiores aos de escolas públicas nos três instrumentos. Quanto à série, embora tenha havido uma melhora no desempenho dos estudantes com a progressão escolar no que se refere à dificuldade de aprendizagem, compreensão em leitura e reconhecimento de palavras, houve diferença estatisticamente significativa somente para este último, o que lhe conferiu uma evidência de validade de critério. 
Em síntese pode-se verificar que os estudos mencionados têm apontado que tanto o Reconhecimento de Palavras, quanto o Cloze foram sensíveis para diferenciar crianças com dificuldade de aprendizagem média e acentuada daquelas com dificuldade leve ou sem indícios de dificuldade. Ao lado disso, também têm evidenciado correlação positiva e significativa entre os acertos no Cloze e do Reconhecimento de Palavras e correlações negativas e significativas entre os erros do Adape e o Reconhecimento de Palavras e entre o Adape e o Cloze.

Considerando a associação entre compreensão em leitura e escrita, tal qual apontado pelos estudos descritos, bem como que aprender a ler e a escrever deixou de ser privilégio dos mais abastados para se tornar uma necessidade e preocupação de todos, parece natural que a necessidade de se detectar com maior precisão os problemas específicos do estudante brasileiro com relação a essas habilidades tenha aumentado na mesma proporção. Essas constatações reforçam a necessidade de instrumentos que possibilitem interpretações mais seguras e coerentes com a realidade vivenciada. Foi com esse intuito que o presente estudo foi proposto. Seu objetivo foi buscar evidências de validade entre o Teste de Cloze e a EAVE, instrumentos que se prestam, respectivamente, à avaliação dos construtos compreensão em leitura e aprendizagem da escrita.

\section{Método}

\section{Participantes}

Participaram deste estudo 221 crianças, ambos os sexos, entre 6 e 12 anos ( $M=8,53$; $D P=1,40)$ de primeira a quarta séries do ensino fundamental de uma escola pública do interior de São Paulo. A maioria dos estudantes era do sexo masculino $(n=120$; $54,3 \%)$, sendo que $56(25,3 \%)$ estudantes frequentavam a primeira série, $54(24,4 \%)$ a segunda, 55 $(24,9 \%)$ a terceira e $56(25,3 \%)$ a quarta série.

\section{Instrumentos}

Questões de identificação - Os sujeitos informaram, na mesma folha da Técnica de Cloze, os seguintes aspectos: nome, idade, sexo e série a qual pertencem.

Teste de Cloze - dois textos, de 204 palavras, "A Princesa e o Fantasma" e "Uma Vingança Infeliz"
(Santos, 2005), de tamanho equivalente, porém níveis de dificuldade diferentes, que foram preparados segundo os padrões tradicionais do teste de Cloze. Tal como aqui utilizado, o Cloze refere-se à estruturação de um texto, do qual se omite todo quinto vocábulo, que é substituído por um traço de tamanho equivalente ao da palavra omitida. 0 respondente deverá completar as lacunas com a palavra que julgar mais adequada para completar o sentido do texto.

A correção foi realizada com base na proposta de correção literal (Bitar, 1989), que considera corretas somente as respostas que contêm palavras idênticas às omitidas. A pontuação máxima possível tanto para o primeiro cloze, "A Princesa e o Fantasma", quanto para o segundo, "Uma Vingança Infeliz", é 15 pontos, número correspondente à quantidade de lacunas contidas nos textos, perfazendo um total de 30 pontos possíveis para a técnica de Cloze. As duas histórias do Cloze que foram utilizadas no presente estudo já estão validadas como medidas de compreensão de leitura (Santos, 2005).

Escala de Avaliação da Escrita (EAVE) (Sisto, 2005) - visa a avaliar a dificuldade de representação de fonemas, ou seja, de grafar letras e palavras a partir de um sistema linguístico estruturado e arbitrário. Consiste no ditado de 55 palavras, sendo que 42 delas apresentam algum tipo de dificuldade classificada como encontro consonantal (lt, $\mathrm{mp}$, nd, $\mathrm{nt}, \mathrm{rc}, \mathrm{rs}, \mathrm{rt}, \mathrm{st}$ ), dígrafo (ch, lh, nh, qu, rr, ss), sílaba composta (br, dr, gr, tr) e sílaba complexa (ao, ci, sa). Foi atribuído um ponto para cada erro e zero para cada acerto cometido pelas crianças no ditado das palavras de acordo com os seguintes critérios de correção, cada palavra foi considerada uma unidade: houve uma contagem dos erros de ortografia, das ausências de palavras, da acentuação errada e do uso indevido de letras maiúsculas e minúsculas, totalizando 55 pontos possíveis.

\section{Procedimento}

Após a autorização das instituições focalizadas e a aprovação do Comitê de Ética, os instrumentos foram aplicados coletivamente, em horário de aula previamente cedido pelo professor. Foi perguntado à professora ou responsável direta pelas turmas se havia crianças que apresentavam alguma alteração perceptual, sensorial, visual ou auditiva importante que seria impeditiva para a realização das atividades propostas. Nesses casos o protocolo foi assinalado e excluído. 
Inicialmente, as crianças cujos pais assinaram o Termo de Consentimento Livre e Esclarecido, preencheram as questões de identificação do sujeito. Em seguida foram solicitadas a lerem cada um dos textos que lhes foram entregues e a preencher as lacunas com as palavras que melhor se adequassem ao contexto. Feito isso, a professora da classe explicou à turma que iria efetuar o ditado de algumas palavras, dizendo que daria um tempo para que as crianças escrevessem uma palavra ao lado da outra, deixando um espaço entre elas, e que as palavras seriam ditadas uma a uma e não seriam repetidas. A professora foi orientada ainda, a solicitar que os alunos não repetissem a palavra ditada e a escrevessem como a compreenderam, além de ressaltar que quando a palavra começasse com letra maiúscula ela diria. Após as devidas explicações, a professora iniciou o ditado das palavras da Escala de Avaliação da Escrita (EAVE), de forma sistemática, palavra por palavra, tendo em vista a familiaridade das crianças com sua entonação de voz.

\section{Resultados e discussão}

Os resultados foram analisados considerando-se os objetivos propostos, de identificar evidências de validade para os instrumentos que avaliam habilidades linguísticas, mais especificamente a compreensão da leitura por meio do Teste de Cloze e o desempenho em escrita pela Escala de Avaliação da Escrita (EAVE). Importante destacar que para esse instrumento não havia, até então, estudos sobre as suas evidências de validade, visto que embora a escala seja derivada do ditado Adape, sobre o qual vários estudos foram realizados, sobre esse instrumento mais simples, fácil de aplicar e corrigir não havia ainda estudos sistemáticos.

Assim sendo, para verificar se a hipótese de que as medidas aqui focalizadas apresentavam evidências de validade baseadas em testes avaliando construtos relacionados, recorreu-se à prova de Correlação de Pearson. Os resultados da análise mostraram uma correlação negativa ou inversa de $r=-0,84(p<0,001)$ entre o EAVE e o Cloze, mostrando que quando a pontuação no EAVE aumenta, a compreensão em leitura diminui. Esses achados indicaram, portanto, a existência de uma forte correlação (Dancey \& Reidy, 2006) entre a aprendizagem da escrita e a compreensão em leitura, que pode ser considerada como excelente segundo os parâmetros de Prieto e Muniz (2000).
Tais resultados comprovaram a relação estreita entre os construtos avaliados e confirmam o fato de que, embora haja uma visão integradora da leitura e da escrita desde meados da década de 1980, a aprendizagem dessas habilidades instrumentais envolve particularidades e, portanto, processos distintos (Santos, 2004). A leitura parte da informação visual e chega ao som, envolvendo a decodificação, enquanto que a escrita se inicia a partir dos segmentos fonológicos e da associação dos fonemas às letras constituindo-se, assim, num processo que abarca predominantemente a codificação (Capovilla \& Capovilla, 2000; Coll, Palacios e Marchres, 1995; Garcia, 1998, Salles \& Parente, 2002).

Embora tenham utilizado um instrumento que avalia a dificuldade de aprendizagem da escrita, estudos como os de Zucoloto e Sisto (2002), Santos et al. (2006) e Cunha (2006) confirmam a relação existente entre a aprendizagem da leitura e da escrita. Assim, entende-se que o alto índice de correlação observado entre a compreensão em leitura e a aprendizagem da escrita no presente estudo não evidencia que ambos os instrumentos estejam medindo construtos idênticos, mas sim construtos que têm muitos elementos em comum.

Também Santos et al. (2006) observaram, a exemplo deste estudo, que os escores do instrumento de escrita utilizado, o Adape, se correlacionaram negativamente com o número de acertos do Cloze. Do mesmo modo, Cunha (2006) encontrou correlações negativas e significativas entre os erros do Adape e o Reconhecimento de Palavras e entre os erros do Adape e os acertos no Cloze.

Considerando-se que o presente estudo não fez uso de medidas de inteligência e, ainda no sentido de analisar melhor a relação existente entre tais construtos, recorreu-se à correlação parcial para verificar o efeito maturacional que poderia estar influenciando o índice de correlação encontrado. Esses dados são apresentados na Tabela a seguir.

Tabela 1 - Correlação parcial (nivel de significância de 0,01) entre as pontuaçōes totais obtidas nas medidas adotadas com controle de variáveis ( $\mathrm{N}=221$ )

\begin{tabular}{cccc}
\hline $\begin{array}{c}\text { Variável } \\
\text { controlada }\end{array}$ & Instrumento & $\begin{array}{r}\text { Correlação } \\
\text { de Pearson }\end{array}$ & Cloze \\
\hline Idade & EAVE & $r$ & $-0,79\left(^{* *}\right)$ \\
Série & EAVE & $r$ & $-0,73\left(^{* *}\right)$ \\
Sexo & EAVE & $r$ & $-0,83\left(^{* *}\right)$ \\
\hline
\end{tabular}

$\left({ }^{* *}\right) \mathrm{p}<0,001$ 
Ao se controlar a idade dos participantes na análise das medidas adotadas, observou-se que a correlação foi significativa e manteve a mesma tendência apresentada quando o efeito dessa variável não havia sido retirado, embora tenha sofrido redução ao se retirar seu efeito. Deste modo, verificou-se que a associação entre os construtos compreensão em leitura e aprendizagem da escrita, se dava parcialmente graças à idade das crianças que compuseram a amostra deste estudo.

Tal qual observado com relação à idade, verificou-se que, no caso da série, a correlação foi significativa e manteve a mesma tendência apresentada anteriormente. 0 resultado com relação ao controle da série evidenciou que ela também influenciou a associação encontrada entre os instrumentos empregados. No entanto, esse resultado parece indicar que o efeito da série sobre os construtos aqui estudados foi ainda maior que o da idade, uma vez que o índice de correlação obtido diminuiu ainda mais quando se controlou o efeito dessa variável $(r=-0,84$ e $r=-0,73$, respectivamente).

No mesmo sentido, a correlação entre os instrumentos empregados manteve as mesmas tendências apresentadas anteriormente, inclusive no que se refere ao seu nível de significância. Quase não houve alteração no índice de correlação entre a compreensão em leitura e a aprendizagem da escrita ao se retirar o efeito da variável sexo, não causando mudança na intensidade da magnitude obtida. Logo, na amostra pesquisada, a associação entre compreensão em leitura e aprendizagem da escrita parece não ter sofrido influência relevante dessa variável. Esses achados confirmam que, conforme ressaltado por diversos autores o nível escolar e a idade das crianças são variáveis importantes na avaliação dessas habilidades (Bradley \& Bryant, 1983; Capovilla \& Capovilla, 1998; Capovilla et al., 2007; Maluf \& Barrera, 1997; Oliveira et al., 2007; Pestun, 2005; Suehiro \& Santos, 2005).

Pode-se afirmar, portanto, que por meio deste estudo mais um instrumento pode ser incluído no rol daqueles que fornecem uma informação válida e confiável para a interpretação do desempenho tanto em compreensão em leitura como do desempenho na aquisição da escrita. Dessa forma, os resultados aqui obtidos reiteram o que foi demonstrado em outros estudos brasileiros que investigaram a relação entre os processos de leitura e escrita (Cunha, 2006; Santos et al., 2006; Zucoloto \& Sisto, 2002).

\section{Considerações finais}

É sabido que a aprendizagem da leitura e da escrita e, de maneira mais ampla, a alfabetização, se constitui num grande desafio do mundo moderno, no qual a incoerência impera. Se por um lado, os recursos tecnológicos ganham diariamente uma dimensão cada vez maior, oferecendo a sociedade recursos como a utilização do computador e a expansão da internet, a fim de facilitar a busca pelo conhecimento, tornando o caminho menos árduo e mais motivador. Por outro, as notícias acerca do desempenho das crianças brasileiras em relação à aprendizagem de habilidades básicas, tais como, a leitura e a escrita são desoladoras.

Diante desse contexto a construção e o desenvolvimento de instrumentos, pautados em princípios científicos, que auxiliem na identificação das dificuldades encontradas por essas crianças se faz imprescindível. Daí a relevância de estudos como o aqui realizado, cujo objetivo foi buscar por evidência de validade concorrente entre instrumentos que se prestam a avaliação das habilidades mais solicitadas em ambientes formais e informais de aprendizagem. Nesse sentido, há que se destacar que a avaliação da compreensão em leitura e da aprendizagem da escrita com base em instrumentos válidos possibilita, além de uma inferência mais confiável quanto aos desempenhos obtidos, que estratégias mais adequadas para sanar os problemas detectados sejam implementadas.

Diversas pesquisas realizadas anteriormente com base no teste de Cloze e no Adape, instrumento que derivou o EAVE, já haviam demonstrado a relação estreita que existe entre a compreensão em leitura e a aprendizagem da escrita. Os resultados aqui encontrados não apenas confirmam os achados de tais estudos, como afirmam a possibilidade de utilização de outros instrumentos, ampliando, dessa forma, o leque de possibilidades de avaliação dos construtos focalizados. Vale destacar, entretanto, que embora este estudo tenha apresentado evidências de validade da EAVE, novas pesquisas sobre outras evidências de validade são necessárias para propiciar a ampliação do conhecimento sobre as suas características psicométricas.

Espera-se ainda que a realização de outras pesquisas venha a sanar as limitações deste estudo, utilizando uma amostra maior, bem como crianças de instituições de natureza jurídica diferentes, a saber, 
escolas públicas e particulares e excluir crianças com defasagens. Ao lado disso, tais estudos poderiam incluir medidas de controle da inteligência a fim de verificar a relação desse construto com os aqui estudados. Ao considerar essas sugestões, essas pesquisas poderão contribuir não apenas para a ampliação dos conhecimentos na área, mas, sobretudo, para o aprimoramento dos instrumentos e procedimentos técnicos empregados na avaliação psicológica.

\section{Referências}

Abraham, R. G., \& Chapelle, C. A. (1992). The meaning of cloze test scores: An item difficulty perspective. The Modern Language Journal, 76(4), 468-479.

Ashby-Davis, C. (1985). Cloze and reading cromprehension: A qualitative analysis and critique. Jounal of Reading, 28(7), 585-589.

Bitar, M. L. (1989). Eficiência dos instrumentos de avaliação em leitura. Dissertação de Mestrado, Pontifícia Universidade Católica de São Paulo, São Paulo.

Bradley, L., \& Bryant, P. E. (1983). Categorizing sounds and learning to read: A causal connection. Nature, 301, 419-421.

Capovilla, A. G. S., \& Capovilla, F. C. (2000). Problemas de leitura e escrita. São Paulo: Memnon.

Capovilla, A. G. S., Dias, N. M., \& Montiel, J. M. (2007). Desenvolvimento dos componentes da consciencia fonológica no ensino fundamental e correlação com nota escolar. Psico-USF, 12(1), 55-64.

Carelli, A. E. (1992). Teste da eficiência de programas de compreensão e leitura crítica. Dissertação de Mestrado, Pontifícia Universidade Católica de Campinas, Campinas.

Coll, C., Palacios, J., \& Marchesi, A. (1995). Desenvolvimento psicológico e educação: Necessidades educativas especiais e aprendizagem escolar. Porto Alegre: Artes Médicas.

Cunha, N. B. (2006). Instrumentos para avaliação da leitura e escrita: Estudos de validade. Tese de Doutorado. Universidade São Francisco, Itatiba.
Cunha, N. B., Suehiro, A. C. B., Oliveira, E. Z., Pacanaro, S. V., \& Santos, A. A. A. (2009). Produção científica da avaliação da leitura no contexto escolar. Revista Psico, 40(1), 17-23.

Dancey, C. P., \& Reidy, J. (2006). Análise de correlação: O r de Pearson. In C. P. Dancey \& J. Reidy (Org.). Estatística sem matemática para a psicologia: Usando SPSS para Windows (pp. 178-218). Porto Alegre: Artmed.

Garcia, J. N. (1998). Manual de dificuldades de aprendizagem: Linguagem, leitura, escrita e matemática. Porto Alegre: Artes Médicas.

Giordano, G. (1985). Learning to reading erased text. Academic Therapy, 20(8), 317-322.

Joly, M. C. R. A., \& Lomônaco, J. F. B. (2003). Avaliando a compreensão de leitura no ensino fundamental: uma comparação entre o instrumento eletrônico e o impresso. Boletim de Psicologia, 53(119), 131-147.

Maluf, M. R., \& Barrera, S. D. (1997). Consciência fonológica e linguagem escrita em pré-escolares. Psicologia: Reflexão e Crítica, 10(1), 125-145.

Noronha, A. P. P. (2002). Problemas mais graves e mais frequentes no uso dos testes psicológicos. Psicologia: Reflexão e Crítica, 15(1), 135-142.

Oliveira, K. L., Boruchovitch, E., \& Santos, A. A. A. (2007). Compreensão de leitura em alunos de sétima e oitava séries do ensino fundamental. Psicologia Escolar e Educacional, 11(1), 41-49.

Oliveira, K. L., Boruchovitch, E., \& Santos, A. A. A. (2009). A técnica de cloze na avaliação da compreensão em leitura. In A. A. A Santos, E. Boruchovitch \& K. L. Oliveira (2009). Cloze: Um instrumento de diagnóstico e intervenção (pp. 47-77). São Paulo: Casa do Psicólogo.

Pestun, M. S. V. (2005). Consciência fonológica no início da escolarização e o desempenho ulterior em leitura e escrita: estudo correlacional. Estudos de Psicologia, 10(3), 407-412.

Poersch, J. M. (1993). Pontos de convergência entre a leitura e escritura. Porto Alegre: EDIPUCRS.

Prieto, G., \& Muñiz, J. (2000). Un modelo para evaluar la calidad de los testes utilizados en españa. Papeles del Psicólogo, 77, 65-75. 
Salles, J. F., \& Parente, M. A. M. P. (2002). Processos cognitivos na leitura de palavras em crianças: Relações com compreensão e tempo de leitura. Psicologia: Reflexão e Crítica, 15(2), 321-331.

Santos, A. A. A. (1994). Programas de remediação: Uma alternativa para o desenvolvimento da compreensão em leitores adultos. Pro-Posições, 5(1-13), 115-122.

Santos, A. A. A. (2004). O Cloze como técnica de diagnóstico e remediação da compreensão em leitura. Interação em Psicologia, 8(2), 217-226.

Santos, A. A. A. (2005). 0 Teste de Cloze como instrumento de diagnóstico e de desenvolvimento da compreensão em leitura, relatório técnico. Itatiba: Universidade São Francisco.

Santos, A. A. A., Sampaio, I. S., Lukjanenko, M. F. S., Cunha, N. B., \& Zenorini, R. P. C. (2006). Avaliação de dificuldades em compreensão de leitura e escrita. In C. Machado, L. S. Almeida, M. Gonçalves \& V. Ramalho (Org.). XI Conferência Internacional de Avaliação Psicológica: Formas e Contextos (pp. 529-536). Braga: Psiquilíbrios.

Santos, A. A. A., Boruchovitch, E., \& Oliveira, K. L. (2009). Apresentação. In A. A. A Santos, E. Boruchovitch \& K. L. Oliveira (2009). Cloze: Um instrumento de diagnóstico e intervenção (pp. 13-19). São Paulo: Casa do Psicólogo.
Sisto, F. F. (2001). Dificuldade de aprendizagem em escrita: Um instrumento de avaliação (Adape). In F. F. Sisto, E. Boruchovitch, L. D. T. Fini, R. P. Brenelli \& S. C. Martinelli (Org.). Dificuldades de aprendizagem no contexto psicopedagógico (pp. 190-213). Petrópolis: Vozes.

Sisto, F. F. (2005). Escala de avaliação da escrita (EAVE), relatório técnico. Itatiba: Universidade São Francisco.

Sisto, F. F., Codenotti, N., Costa, C. A. J., \& Nascimento, T. C. N. (1979). Testes psicológicos no Brasil: que medem realmente. Educação e Sociedade, 2(2), 152-165.

Sousa, E. O. (2005). Habilidades metassistáticas e aprendizagem da leitura: Estudo com crianças da $1^{a}$ a série do ensino fundamental. Tese de Doutorado, Pontifícia Universidade Católica de São Paulo, São Paulo.

Suehiro, A. C. B., \& Santos, A. A. A. (2005). O Bender e as dificuldades de aprendizagem: Estudo de validade. Avaliação Psicológica, 4(1), 23-31.

Taylor, W. L. (1953). Cloze procedure: A new tool for measuring readability. Journalism Quarterly, 30, 415-433.

Zucoloto, K. A., \& Sisto, F. F. (2002). Dificuldades de aprendizagem em escrita e compreensão em leitura. Interação, 6(2), 157-166. 